\title{
Analysis of macular cone photoreceptors in a case of occult macular dystrophy
}

This article was published in the following Dove Press journal:

Clinical Ophthalmology

7 May 2013

Number of times this article has been viewed

\section{Naoki Tojo \\ Tomoko Nakamura \\ Hironori Ozaki \\ Miyako Oka \\ Toshihiko Oiwake \\ Atsushi Hayashi}

Department of Ophthalmology, University of Toyama, Toyama, Japan
Correspondence: Atsushi Hayashi Department of Ophthalmology, Graduate School of Medicine and Pharmaceutical Sciences, University of Toyama, 2630 Sugitani, Toyama 930-0194, Japan Tel +8I 764347363

Fax +8I 764345037

Email ganka@med.u-toyama.ac.jp
Purpose: To investigate changes in cone photoreceptors with adaptive optics (AO) fundus imaging and spectral domain optical coherence tomography (SD-OCT) in a case of occult macular dystrophy (OMD).

Patient and methods: Both eyes of a 42-year-old woman diagnosed with OMD were examined. We used an $\mathrm{AO}$ fundus camera to obtain images of cone photoreceptors in the macula of the OMD subject and five healthy control subjects. Correlations between the AO images and the SD-OCT images were examined. Cone photoreceptors in eight areas in the macula of OMD and healthy control subjects were analyzed and compared.

Results: SD-OCT showed a loss of the cone outer-segment tips line outside of the fovea in both eyes of the subject with OMD. The left eye with decreased visual acuity showed a discontinuous photoreceptor inner-segment and outer-segment line and cone outer-segment tips line at the fovea in SD-OCT and loss of cone mosaics as a dark spot in the AO image. In panoramic AO images and cone-density maps, less cone density was observed in a ring-like region outside the fovea than in the peripheral retina. In most of the areas examined, the cone densities were lower in the OMD eyes than in the healthy control eyes.

Conclusions: Cone densities in the macula of the OMD patient were greatly decreased. AO images were found to be useful to evaluate morphologic changes in cone photoreceptors in patients with OMD.

Keywords: occult macular dystrophy, adaptive optics, cone photoreceptor, cone analysis, optical coherence tomography

\section{Introduction}

Occult macular dystrophy (OMD) is an unusual, inherited macular dystrophy characterized by essentially normal appearances of the fundus and fluorescein angiography, with progressive decrease of visual acuity in both eyes. ${ }^{1}$ These patients have normal full-field electroretinograms (ERGs), but show severely decreased macular responses by multifocal ERG. ${ }^{2}$ It has been emphasized that the main key to differentiate OMD from other diseases, such as optic neuritis, dominant optic atrophy, amblyopia, or psychological disorders, is the multifocal ERG from the central retina.

Recently, newly developed ophthalmologic instruments have allowed us to investigate the details of retinal morphology in vivo. For example, spectral domain optical coherence tomography (SD-OCT) is a well-established method to examine the layers of retinal architecture. ${ }^{3}$ Decreases in foveal thickness and disturbances of the photoreceptor inner- and outer-segment junction (IS/OS) have been observed by SD-OCT in patients with OMD. ${ }^{4}$ The adaptive optics (AO) system can compensate 
for optical aberrations and provide high-resolution retinal images, such as images of cone photoreceptors and retinal vessels. ${ }^{5}$ We and others have demonstrated the feasibility of using an $\mathrm{AO}$ system to image cone photoreceptors in the macular degeneration of patients. ${ }^{6-8}$

Because the pathophysiology of OMD is not well understood, in the present study we examined correlations between images of SD-OCT and AO in a case with OMD and analyzed cone photoreceptors in the AO images using cone-analysis software.

\section{Methods}

The patient underwent complete ophthalmic examinations at Toyama University Hospital, which included a fundus examination, fluorescein angiography, scotopic and photopic full-field ERG, multifocal ERG, SD-OCT, and AO fundus images. Informed consent was obtained after a full explanation of the procedures. All studies were conducted in accordance with the principles embodied in the Declaration of Helsinki. The study protocol for the AO fundus camera was approved by the institutional review board of the University of Toyama.

The diagnosis of OMD was made based on the following findings: normal appearance of the fundus by fundus photography and fluorescein angiography, decreased visual acuity, normal responses of scotopic and photopic full-field ERGs, and reduced amplitudes of multifocal ERG.

The scotopic and photopic full-field ERGs were recorded with an LE-4000 system (Tomey, Nagoya, Japan), and the multifocal ERG was recorded with an LE4100 system (Mayo, Inazawa, Japan), according to the International Society for Clinical Electrophysiology of Vision standards. The stimulus array consisted of 61 hexagons, and the luminance of each hexagon was alternated between $200 \mathrm{~cd} / \mathrm{m}^{2}$ and $5 \mathrm{~cd} / \mathrm{m}^{2}$ with a $75 \mathrm{~Hz}$ frame rate. The retinal morphology was investigated by SD-OCT (RS-3000; Nidek, Gamagori, Japan). Fluorescein angiography was performed with an F-10 scanning laser ophthalmoscope (Nidek). After the pupil was dilated with an eye drop of $0.5 \%$ toropicamide and $0.5 \%$ phenylephrine, AO images of both eyes were taken with an AO fundus camera (RTX1; Imagine Eyes, Orsay, France). The AO images were analyzed with the original coneanalysis software (AODetect; Imagine Eyes) created by the manufacturer. ${ }^{7}$ We analyzed the cone density, cone spacing, and mosaic regularity of cone photoreceptors at $500,1000,2000$, and $3000 \mathrm{~mm}$ from the foveal center in the temporal or nasal retina and compared them to the data of healthy control eyes. AO images of five eyes of five healthy control subjects were taken with the RTX1 system and analyzed with the software provided by the manufacturer.

Genetic testing for the retinitis pigmentosa 1-like 1 (RP1L1) gene was performed using the blood sample at Nippon Medical School Chiba Hokusoh Hospital as previously described. ${ }^{9}$

\section{Case report}

The patient was a 42-year-old woman who did not report a family history of similar visual problems. The best-corrected decimal visual acuity was 1.0 in the right eye and 0.2 in the left eye by a conventional Landolt $\mathrm{C}$ chart. Color fundus photographs and fluorescein angiography showed normal findings in both eyes (Figure 1). Multifocal ERG showed reduced amplitudes in the central ring of both eyes (right eye $25.62 \mathrm{nV} / \mathrm{deg}^{2}$, left eye $11.80 \mathrm{nV} / \mathrm{deg}^{2}$ ) (Figure 2A, B, D and E), while scotopic and photopic full-field ERGs showed normal responses (Figure $2 \mathrm{C}$ and $\mathrm{F}$ ). The responses of the central eight areas of multifocal ERG (circles in Figure 2A and D) showed a similar decrease between the nasal and the temporal retina in both eyes. An SD-OCT image revealed a relatively normal IS/OS line and cone outer-segment tips (COST) line at the fovea in the right eye (arrowheads in Figure 3A), with a reduced IS/OS line and unclear COST line in the outside of the fovea (arrows in Figure 3A). A panoramic AO image of the right eye $(6.25 \times 0.95 \mathrm{~mm})$ showed an extensive loss of cone photoreceptors in the entire area examined, with a ring-like area of residual cone photoreceptors at the fovea (arrowheads in Figure 3B). However, cone photoreceptors were observed at the foveal center (arrow in Figure 3B). An SD-OCT image revealed a discontinuous IS/OS line and COST line at the foveal center in the left eye (asterisk
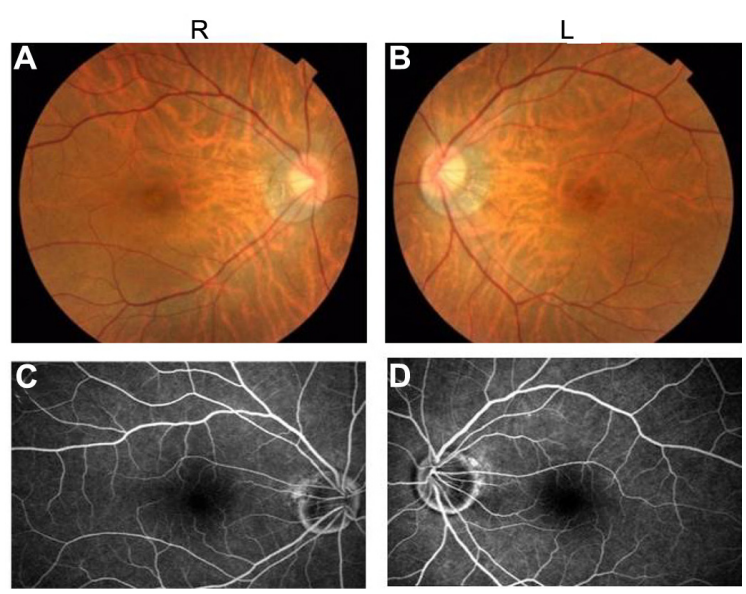

Figure I Photographs of the fundus (A and B) and fluorescein angiography (C and $\mathbf{D}$ ) of both eyes of a patient with occult macular dystrophy. Abbreviations: $R$, right eye; $L$, left eye. 


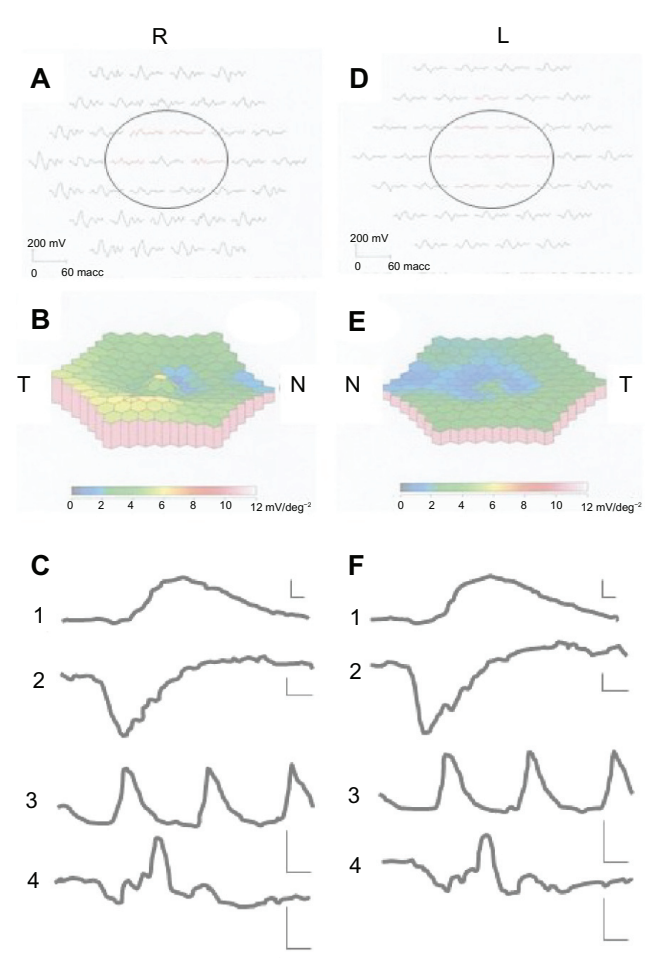

Figure 2 Images of multifocal electroretinograms (ERGs) (A, B, D and E), and full-field ERG ( $\mathbf{C}$ and $\mathbf{F}$ ). In the full-field ERG ( $\mathbf{C}$ and $\mathbf{F}$ ), I shows the graph for the rod ERG, 2 that for the flash ERG, 3 that for the $30 \mathrm{~Hz}$ flicker ERG, and 4 that for the cone ERG. Note: The circles of $A$ and $D$ show the response of the central 8 areas. The scales ( $\mathrm{C}$ and $\mathrm{F}$ ) represent $10 \mathrm{~ms}$ (horizontal axis) and $100 \mu \mathrm{V}$ (vertical axis). Abbreviations: R, right eye; L, left eye; $N$, nasal; $T$, temporal.

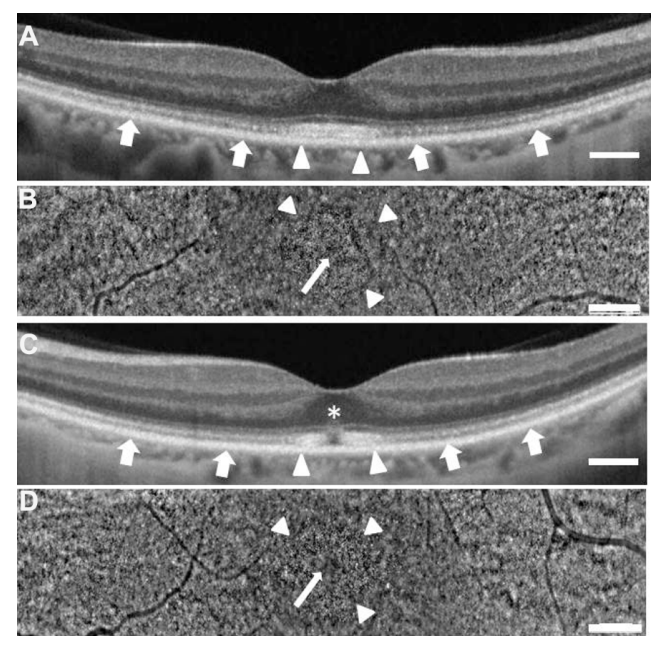

Figure 3 Images of spectral domain optical coherence tomography (A and $\mathbf{C})$ and adaptive optics (B and $\mathbf{D}$ ) at the fovea of the OMD patient.

Notes: Images for the right eye are shown in $\mathbf{A}$ and $\mathbf{B}$, and those for the left eye are presented in $\mathbf{C}$ and $\mathbf{D}$. Panoramic adaptive optics images of $\mathbf{B}$ the right eye $(6.25 \times 0.95 \mathrm{~mm})$, and $\mathbf{D}$ the left eye $(6.0 \times 1.04 \mathrm{~mm})$. The arrowheads in $A$ and $C$ show areas of observable IS/OS line and COST line at the fovea. The arrowheads in $B$ and $D$ show the corresponding areas in $A O$ images. The long arrow in $B$ and $D$ shows foveal center. The short arrows in $A$ and $C$ show the areas with a reduced IS/OS line and unclear COST line. An asterisk in $C$ shows defect of IS/OS line and COST line. Bar $=500 \mu \mathrm{m}$.

Abbreviations: OMD, occult macular dystrophy; IS/OS, inner- and outer-segment; COST, cone outer-segment tips; $\mathrm{AO}$, adaptive optics. in Figure 3C). Similar to the findings in the right eye, the IS/OS line and the COST line in the left eye were observed at the fovea (arrowheads in Figure 3C), with a reduced IS/ OS line and unclear COST line in the outside of the fovea (arrows in Figure 3C). A panoramic AO image of the left eye $(6.0 \times 1.04 \mathrm{~mm})$ showed an extensive loss of cone photoreceptors in the entire area, with a ring-like region of residual cone photoreceptors at the fovea (arrowheads in Figure 3D). A dark area was observed at the foveal center without cone mosaics (arrow in Figure 3D), and this area corresponded with the area exhibiting loss of the IS/OS line and COST line in the SD-OCT image (asterisk in Figure 3C). The central macular thickness in the SD-OCT images was $148 \mu \mathrm{m}$ in the right eye and $161 \mu \mathrm{m}$ in the left eye.

Eight $100 \times 100 \mu \mathrm{m}$ areas at the nasal or temporal retina were selected (avoiding large retinal vessels) in the panoramic $\mathrm{AO}$ images (Figure 4A, C and E). The AO images of these areas were analyzed with the software. The distances of nasal (N) 500 and temporal (T) 500, N1000 and T1000, N2000 and T2000, and N3000 and T3000 were $500 \mu \mathrm{m}, 1000 \mu \mathrm{m}$, $2000 \mu \mathrm{m}$, and $3000 \mu \mathrm{m}$ from the nasal or temporal foveal center, respectively. The eight areas in a panoramic AO image of a healthy control left eye $(6.51 \times 0.96 \mathrm{~mm})$ are shown in Figure 4A, and the corresponding cone-density panoramic map is shown in Figure 4B. The results of the cone analysis at these eight areas of the five healthy control eyes are shown in Table 1 . The data were expressed as the mean \pm standard deviation. As shown in Figure 4B, the cone densities at the foveal center were lower than those at the outside of the foveal center (asterisk in Figure 4B). This was because the resolution of the AO fundus camera was not sufficient to distinguish each cone photoreceptor at the foveal center. Therefore, we did not perform cone analysis at the foveal center in this study.

Both eyes of the OMD case were similarly examined with an AO fundus camera. Eight $100 \times 100 \mu \mathrm{m}$ areas at the nasal or temporal retina were selected (avoiding large retinal vessels) in panoramic $\mathrm{AO}$ images of both eyes. A panoramic AO image $(6.3 \times 1.03 \mathrm{~mm})$ of the right eye is shown in Figure 4C, and the corresponding cone-density panoramic map is shown in Figure 4D. High cone densities were observed at the fovea, while a ring-like area around the fovea showed lower cone densities than the peripheral areas in the nasal or temporal retina (arrowheads in Figure 4D).

A panoramic AO image $(6.51 \times 0.96 \mathrm{~mm})$ of the left eye is shown in Figure 4E, and the corresponding cone-density panoramic map is shown in Figure 4F. Low cone densities were observed at the foveal center, and these densities corresponded with the dark area of the AO image. The left eye showed a ring-like area of low cone densities around the 

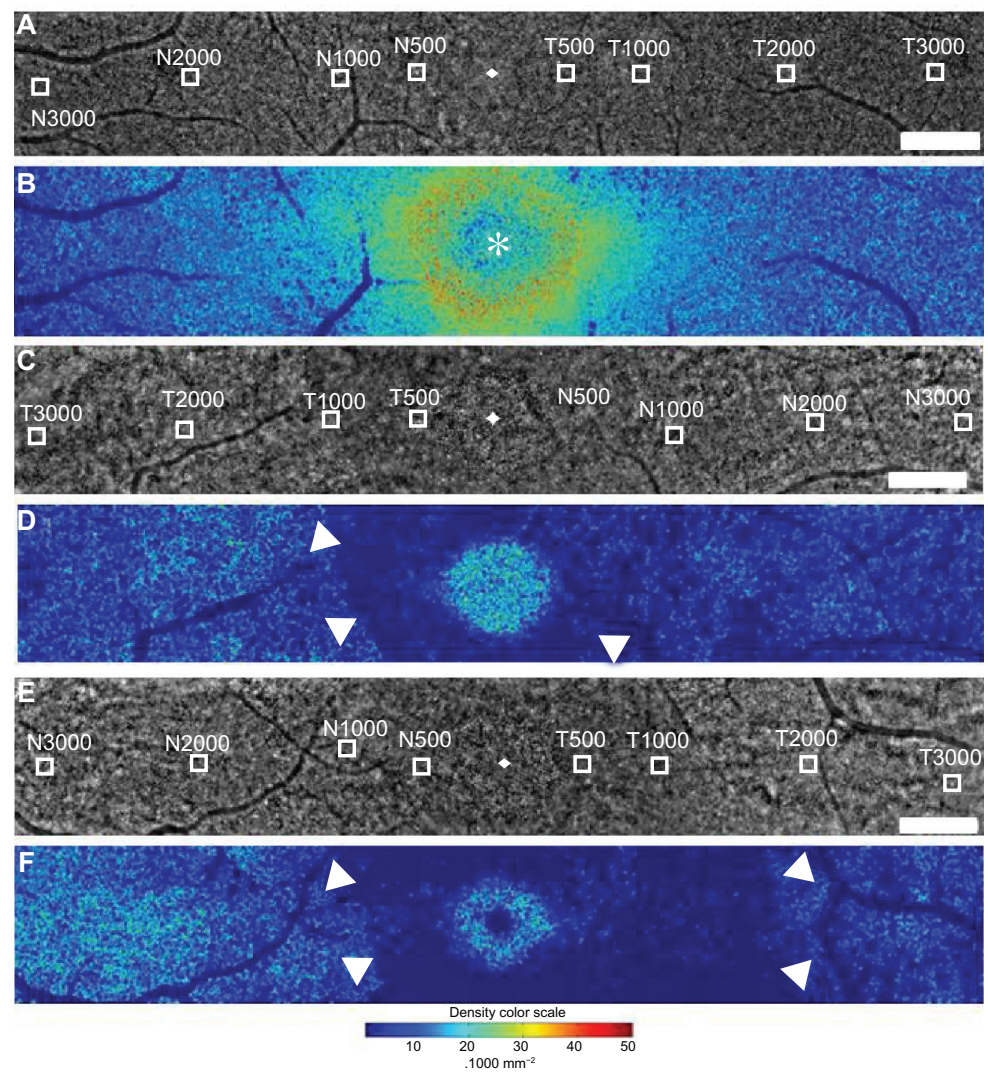

Figure 4 Panoramic adaptive optics images of the left eye of a healthy control $(\mathbf{A}, 6.5 \mathrm{I} \times 0.96 \mathrm{~mm})$, the right eye of the OMD patient $(\mathbf{C}, 6.3 \times 1.03 \mathrm{~mm})$, left eye of the OMD patient $(\mathbf{E}, 6.5 \mathrm{I} \times 0.96 \mathrm{~mm})$, and the corresponding cone-density maps $(\mathbf{B}, \mathbf{D}$ and $\mathbf{F})$, respectively. Eight $100 \times 100 \mu \mathrm{m}$ areas at the nasal or temporal retina were selected $(\mathbf{A}, \mathbf{C}$ and $\mathbf{E})$. Notes: The foveal center is indicated by the asterisk in $\mathbf{B}$. The arrowheads in $\mathrm{D}$ and $\mathrm{F}$ show a ring-like dark area around the fovea with lower cone densities than the peripheral areas in the nasal or temporal retina. Bar $=500 \mu \mathrm{m}$. A color scale of cone density is shown at the bottom.

Abbreviations: OMD, occult macular dystrophy; $\mathrm{N}$, nasal; $\mathrm{T}$, temporal.

fovea, and this area was similar to, but larger than, that of the right eye (arrowheads in Figure 4F).

Eight areas were analyzed with the software, and the color maps and results are shown in Figure 5A (right eye) and B (left eye) and Table 1. In the right eye, cone densities in the OMD patient were lower than those of the healthy control eyes, except in region N3000. In the left eye, the cone densities of the OMD patient were lower than those of the healthy control eyes, except in regions N2000 and N3000. The cone densities in the temporal retina seemed to be lower

Table I Cone analysis of healthy control eyes and both eyes of a patient with OMD

\begin{tabular}{|c|c|c|c|c|c|c|c|c|}
\hline & N500 & NI000 & N2000 & N3000 & T500 & TI000 & T2000 & T3000 \\
\hline \multicolumn{9}{|l|}{ Density $\left(/ \mathrm{mm}^{2}\right)$} \\
\hline Right & 3379 & 6360 & 10733 & 9044 & 1193 & 3478 & 3280 & 6360 \\
\hline Left & 2385 & 5466 & 15305 & 14609 & 2683 & 1689 & 6957 & 5665 \\
\hline $\begin{array}{l}\text { Healthy control } \\
(n=5)\end{array}$ & $2547 I \pm 3829$ & $18505 \pm 2050$ & $|40| 3 \pm 1 \mid 97$ & $10296 \pm 2101$ & $25342 \pm 2754$ & $20475 \pm 1193$ & $14589 \pm 1206$ & $11806 \pm 2208$ \\
\hline \multicolumn{9}{|l|}{ Spacing $(\mu \mathrm{m})$} \\
\hline Right & 17.62 & $|3.4|$ & 10.75 & 11.64 & 27.44 & 117.12 & 17.5 & 12.45 \\
\hline Left & 25.43 & 14.79 & 9.03 & 8.96 & 19.7 & 25.45 & 12.85 & 14.83 \\
\hline $\begin{array}{l}\text { Healthy control } \\
(n=5)\end{array}$ & $6.94 \pm 0.52$ & 8. $18 \pm 0.42$ & $9.32 \pm 0.33$ & $10.88 \pm 0.98$ & $6.96 \pm 0.39$ & $7.81 \pm 0.38$ & $9.10 \pm 0.39$ & $10.29 \pm 0.92$ \\
\hline \multicolumn{9}{|c|}{ Voronoi (\% of six-sided polygons) } \\
\hline Right & 26.5 & 21.9 & 44.4 & 25.3 & 25.0 & 22.1 & 33.3 & 29.7 \\
\hline Left & 33.3 & 30.9 & 38.3 & 31.3 & 29.6 & 23.5 & 28.6 & 31.6 \\
\hline $\begin{array}{l}\text { Healthy control } \\
(n=5)\end{array}$ & $41.7 \pm 4.2$ & $43.9 \pm 2.5$ & $40.3 \pm 4.1$ & $38.1 \pm 4.5$ & $42.6 \pm 4.3$ & $51.6 \pm 2.6$ & $43.2 \pm 6.7$ & $41.6 \pm 3.9$ \\
\hline
\end{tabular}

Note: Data are means \pm standard deviation.

Abbreviations: OMD, occult macular dystrophy; $\mathrm{N}$, nasal; $\mathrm{T}$, temporal. 


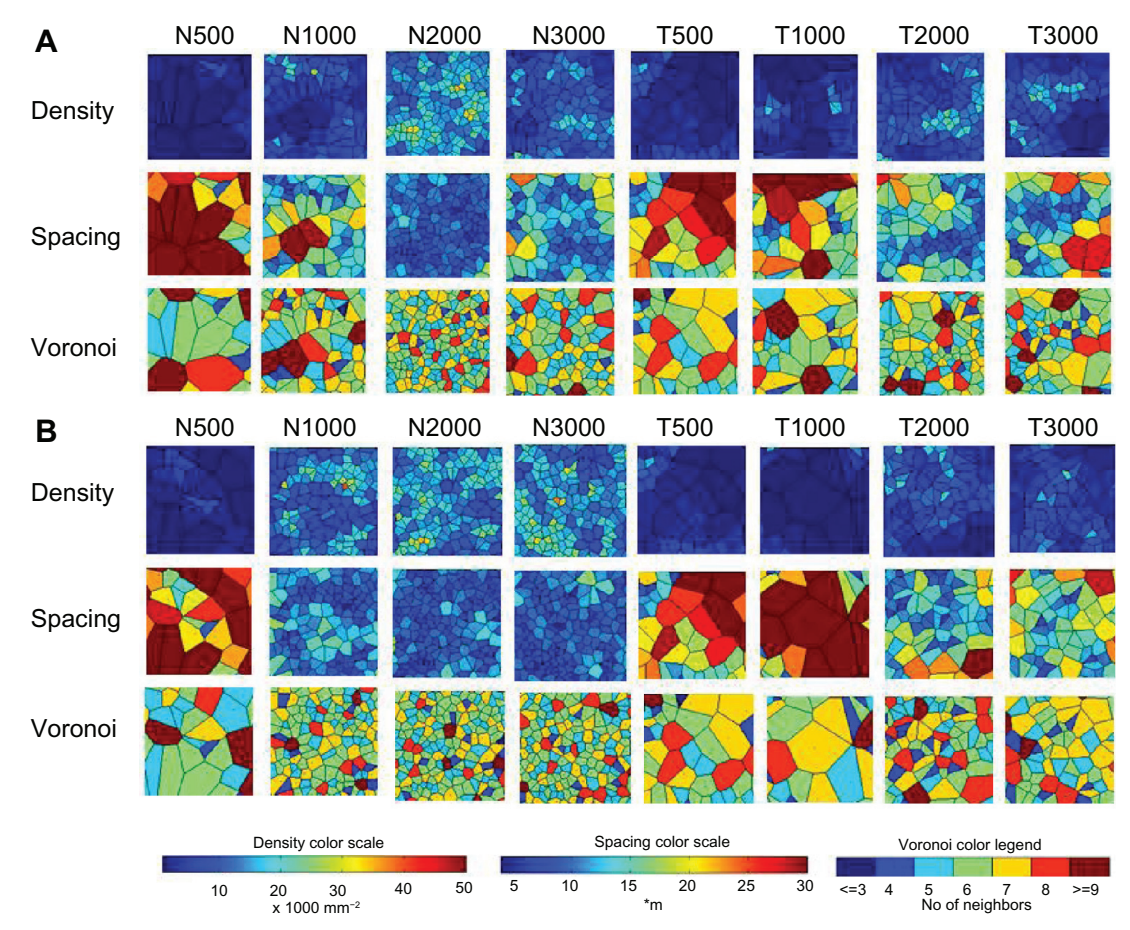

Figure $\mathbf{5}$ Cone analysis in the eight selected areas of the OMD patient. (A right eye, B left eye).

Notes: Color maps of cone density, cone spacing, and Voronoi analysis are shown. Color scales for each analysis are shown at the bottom. Abbreviations: OMD, occult macular dystrophy; $\mathrm{N}$, nasal; $\mathrm{T}$, temporal.

than those in the nasal retina in both eyes. The results of the cone spacing and Voronoi analyses corresponded with those of the cone densities.

In the genetic testing, no mutations in the RPIL1 gene were detected in this case.

\section{Discussion}

OMD is considered to show retinal dysfunctions only in the macula, without apparent changes in ophthalmoscopy and fluorescein angiography. ${ }^{1,2}$ Recently, analyses using SD-OCT revealed detailed retinal structures and abnormal changes in patients with OMD. ${ }^{4,7,10}$ These findings included thinning of the foveal thickness and the outer nuclear layer, ${ }^{3}$ and disruption of the IS/OS line and COST line. ${ }^{7,10}$ Park et al reported that photoreceptor disruption sizes in SD-OCT correlated with visual function and disease progression in OMD. ${ }^{10} \mathrm{We}$ observed similar changes in SD-OCT, but visual function was not analyzed in our case. We also examined the correlations between SD-OCT and AO images, and found that the region with the residual IS/OS line and COST line showed more cone photoreceptors in $\mathrm{AO}$ images than did the regions without a COST line.

Since the pathologic changes seemed to be localized in photoreceptors in OMD, investigation of photoreceptors in vivo is important in this disease. For this purpose, examinations with AO systems seemed suitable. Kitaguchi et al reported that patchy dark areas were detected in all examined eyes of patients with OMD. ${ }^{7}$ However, detailed cone analyses were not performed in the OMD cases. In this study, we examined AO images of healthy control eyes and both eyes of one OMD case with an AO fundus camera, and cone analyses were performed using the cone-analysis software. In our case, lower cone densities were detected around the fovea than in the peripheral retina examined in both eyes, and the areas in the temporal retina showed particularly reduced cone densities relative to those in the nasal retina. Because cone densities in the temporal retina were shown to be slightly lower within the $1 \mathrm{~mm}$ retinal eccentricity than in the nasal retina by histologic and AO studies, ${ }^{11,12}$ cone photoreceptors might be more damaged around the fovea and reduced in the temporal retina than in the nasal retina. In addition, the decreased responses in the central eight areas by multifocal ERG correlated with the decrease in cone densities from N3000 to T3000 in the AO images. We could not examine correlations between the cone densities and the amplitudes and implicit times of the ERG responses in the central eight areas, because we examined only one patient with OMD. The examinations of AO images may be useful to monitor changes in cone photoreceptors in OMD cases.

Our case did not show any mutations in the RP1L1 gene by genetic testing. Because in a previous study, no mutations were detected in four patients with OMD from a single 
Caucasian family, ${ }^{13}$ OMD is considered to be a genetically heterogeneous disorder.

Because this is a case report, we did not perform any statistical analyses of the cone photoreceptors. However, we demonstrated horizontal distributions of cone mosaics and cone densities of one OMD case with an AO fundus camera. In future studies, it will be necessary to statistically analyze the cone photoreceptors of a greater number of OMD cases using AO systems, and to examine longitudinal changes in the cone photoreceptors and correlations between cone analyses and other fundus-imaging findings.

\section{Acknowledgment}

The authors are deeply grateful to Shuhei Kameya, MD, PhD, of Nippon Medical School Chiba Hokusoh Hospital, Chiba, Japan, for performing the genetic testing.

\section{Disclosure}

The authors report no conflicts of interest in this work.

\section{References}

1. Miyake Y, Ichikawa K, Shiose Y, Kawase Y. Hereditary macular dystrophy without visible fundus abnormality. Am J Ophthalmol. 1989;108:292-299.

2. Miyake Y, Horiguchi M, Tomita N, et al. Occult macular dystrophy. Am J Ophthalmol. 1996;122:644-653.

Clinical Ophthalmology

\section{Publish your work in this journal}

Clinical Ophthalmology is an international, peer-reviewed journal covering all subspecialties within ophthalmology. Key topics include: Optometry; Visual science; Pharmacology and drug therapy in eye diseases; Basic Sciences; Primary and Secondary eye care; Patient Safety and Quality of Care Improvements. This journal is indexed on
3. Ooto S, Hangai M, Tomidokoro A, et al. Effects of age, sex, and axial length on the three-dimensional profile of normal macular layer structures. Invest Ophthalmol Vis Sci. 2011;52:8769-8779.

4. Brockhurst RJ, Sandberg MA. Optical coherence tomography findings in occult macular dystrophy. Am J Ophthalmol. 2007;143:516-518.

5. Williams DR. Imaging single cells in the living retina. Vision Res. 2011;51: 1379-1396.

6. Duncan JL, Zhang Y, Gandhi J. High-resolution imaging with adaptive optics in patients with inherited retinal degeneration. Invest Ophthalmol Vis Sci. 2007;48:3283-3291.

7. Kitaguchi Y, Kusaka S, Yamaguchi T, Mihashi T, Fujikado T. Detection of photoreceptor disruption by adaptive optics fundus imaging and Fourier-domain optical coherence tomography in eyes with occult macular dystrophy. Clin Ophthalmol. 2011;5:345-351.

8. Tojo N, Nakamura T, Fuchizawa C, Oiwake T, Hayashi A. Adaptive optics fundus images of cone photoreceptors in the macula of patients with retinitis pigmentosa. Clin Ophthalmol. 2013;7:203-210.

9. Akahori M, Tsunoda K, Miyake Y, et al. Dominant mutations in RP1L1 are responsible for occult macular dystrophy. Am J Hum Genet. 2010;87:424-429.

10. Park SJ, Woo SJ, Park KH, Hwang JM, Chung H. Morphologic photoreceptor abnormality in occult macular dystrophy on spectraldomain optical coherence tomography. Invest Ophthalmol Vis Sci. 2010; 51:3673-3679.

11. Curcio CA, Sloan KR, Kalina RE, Hendrickson AE. Human photoreceptor topography. J Comp Neurol. 1990;292:497-523.

12. Song H, Chui TY, Zhong Z, Elsner AE, Burns SA. Variation of cone photoreceptor packing density with retinal eccentricity and age. Invest Ophthalmol Vis Sci. 2011;52:7376-7384.

13. Chen CJ, Scholl HPN, Birch DG, Iwata T, Miller NR, Goldberg MF. Characterizing the phenotype and genotype of a family with occult macular dystrophy. Arch Ophthalmol. 2012;130:1554-1559.

\section{Dovepress}

PubMed Central and CAS, and is the official journal of The Society of Clinical Ophthalmology (SCO). The manuscript management system is completely online and includes a very quick and fair peer-review system, which is all easy to use. Visit http://www.dovepress.com/ testimonials.php to read real quotes from published authors. 